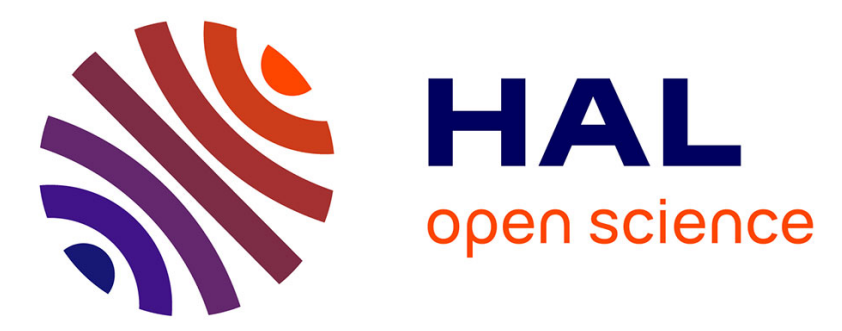

\title{
A robust image-encryption approach against transmission errors in Communicating Things Networks
}

\author{
A. Mostefaoui, Z. Fawaz, H. Noura
}

\section{To cite this version:}

A. Mostefaoui, Z. Fawaz, H. Noura. A robust image-encryption approach against transmission errors in Communicating Things Networks. Ad Hoc Networks, 2019, 94, pp.101947 -. 10.1016/j.adhoc.2019.101947 . hal-03487310

\section{HAL Id: hal-03487310 \\ https://hal.science/hal-03487310}

Submitted on 20 Dec 2021

HAL is a multi-disciplinary open access archive for the deposit and dissemination of scientific research documents, whether they are published or not. The documents may come from teaching and research institutions in France or abroad, or from public or private research centers.
L'archive ouverte pluridisciplinaire HAL, est destinée au dépôt et à la diffusion de documents scientifiques de niveau recherche, publiés ou non, émanant des établissements d'enseignement et de recherche français ou étrangers, des laboratoires publics ou privés.

\section{다)(1) $(5$}

Distributed under a Creative Commons Attribution - NonCommerciall 4.0 International 


\title{
A Robust Image-Encryption Approach Against Transmission errors in Communicating Things Networks.
}

\author{
A. Mostefaouia ${ }^{a}$, Z. Fawaz ${ }^{\text {a }}$ H. Noura ${ }^{\mathrm{b}}$ \\ ${ }^{a}$ FEMTO-ST Institute DISC dep., CNRS UMR 6174, University of Franche Comte \\ Rue Engel Gros, BP 527, 90000, BELFORT, France \\ Email:ahmed.mostefaoui@univ-fcomte.fr,zeinab.fawaz@univ-fcomte.fr \\ ${ }^{b}$ Faculty of Engineering, Lebanese University, Hadath Campus, Lebanon: hnouran@gmail.com
}

\begin{abstract}
In this paper, we target the problem of image transmission in Communicating Things Networks (CTNs) where the ultimate objective is to preserve both the visual quality of the received data at the destination node and the network resources, energy in particular. Nevertheless, visual quality is known to be proportional to the image volume (i.e., better visual quality leads to voluminous data and vice-versa) and hence inversely proportional to the energy cost. Furthermore, as CTNs are open infrastructures, they are prone to transmission errors which are almost managed through costly data re-transmissions. We propose a novel approach, composed of two components: (a) a low layer communication protocol coupled with (b) an image errors recovery filtering mechanisms; which avoids re-transmission due to communication errors. The key idea behind our proposal is to manage errors in transmitted data at the application layer, rather than at the network layer. In other words, corrupted packets are transferred from the lower layers to the upper layers, and a filtering algorithm is in charge to reconstruct the corrupted image. The results of the extensive experiments we conducted have demonstrated the powerful strength of the proposed solution to resist against various types of errors and to enhance the visual quality of images whilst ensuring both data confidentiality and network resources preservation.
\end{abstract}

Keywords:

Voronoi Image Encryption, Confidentiality, Security, Errors Resilience, Noisy networks

\section{Introduction}

In Communicating Things networks (CTNs), a node (i.e., device) is in charge of collecting multimedia data (images) from its immediate physical environment, performing some processing tasks (i.e., coding) with it and finally transmitting it through potentially unreliable wireless communication, to the remote base station for exploitation. Because of the intrinsic characteristics of images (i.e., large volume in comparison to scalar data, high redundancy and strong correlation between adjacent pixels) on one side, and the limitations of the platform in terms of CPU power, memory space and energy provision on the other side, securing images represents a motivating research challenge. Furthermore, the open nature of the platform (i.e., wireless communications) accentuates the need for advanced securing mechanisms that must take these intrinsic limitations into account. Hence, the following requirements have to be considered for any securing approach in the early stages of its design: 
- Data volume: In fact, a communication task is the most expensive task that a node has to execute, since it requires the most energy consumption [1]. For this reason, reducing the amount of transmitted data (i.e, usually proportional to the energy communication cost) prolongs the network lifetime.

- Computing complexity: Sensor devices have limited computing capabilities. Thus, it is necessary to perform a lightweight encryption algorithm, that is efficient in terms of time complexity as well as of space complexity.

- Content confidentiality: Ensuring data confidentiality remains the ultimate goal that has drawn the attraction of many researchers in the CTNs field. In other words, a robust encryption technique must be applied, in order to keep the visual content of the transmitted data hidden for an unauthorized node.

- Errors and active attacks resilience: Because of its intrinsic unreliable communication, CTNs are known to be noisy environments [2]. Thus, it makes the transmitted data vulnerable to active attacks that try to corrupt the transmitted ciphered content. New mechanisms are then necessary to allow the receiver to extract the content of the transmitted data, even when the latter has been corrupted either by noisy communication or by an active attacker.

These requirements are somehow orthogonal and some previous research works have addressed only parts of these requirements and not all (see next section). For instance, the approach presented in [3] has addressed the three first requirements mentioned above. In fact, based on Voronoi tessellation, this approach has demonstrated its efficiency in terms of significant data reduction as well as in terms of data confidentiality preservation. The security results confirmed its robustness against passive attacks. Nevertheless, the issue of resilience to communication errors and active attacks has not been explicitly studied. This issue is particularly important to address in environments prone to frequent communication errors, as those of CTNs. Furthermore, besides the efforts of recent protocols to improve transmission efficiency in terms of energy consumption reduction and increased reliability, they still rely on retransmission of corrupted data. By doing so, an overhead in terms of energy consumption as well as in term of time delivery is generated.

In this paper, we investigate, in addition to previously mentioned requirements, the issue of resilience to frequent transmission errors and active attacks. To this end an based on Voronoi tesselation approach [3], we propose a novel mechanism (new transmission protocol coupled to an image filtering approach) to cope with these two major issues. In fact, instead of relying on retransmission protocols, the receiver could recover the missed or corrupted data through appropriate content processing techniques (i.e., image filtering). Upon receiving noisy data, filtering layer attempts to improve the visual quality of the received corrupted data. Experimental results show a noticeable increase in the perceived quality of the filtered data while significantly reducing the retransmission overhead. The contributions of the proposed mechanism can be resumed by the following points:

- Overhead Reduction: The proposed new communication protocol, inspired from the ARQ protocol [4], has significantly improved the network performance, since it reduces the number of retransmissions. In addition, the proposed filtering mechanisms have, by construction, lower complexity which limits noticeably the introduced overhead.

- Efficient Error Correction: The proposed filtering mechanisms ensure a great enhancement of the visual content for the received corrupted data (i.e., resistance to approximately $75 \%$ of errors).

- Lightweight Scheme: In comparison to state-of-art approaches that either achieve data confidentiality only or achieve data reduction and confidentiality, our proposed scheme has demonstrated its highest strength against different types of perturbations, using an efficient and low complex manner. 
The remainder of the paper is structured as follows: Section 2 presents the related work of this paper. In Section 3, we review the proposed Voronoi-based approach. Then, Section 5.1.2 discusses some parameters that have an important influence on the proposed approach. After that, Section 4 presents the new communication protocol, and investigates the novel filtering mechanisms of error correction at the destination node. Section 5 evaluates the performance of our proposed approach, and studies its error resiliency before and after applying the novel filtering techniques. Section 6 concludes the paper.

\section{Related Work}

Because of the intrinsic characteristics of images (bulk data capacity, high spacial redundancy and strong correlation between adjacent pixels [5]), on one hand and the numerous constraints of Wireless Multimedia Sensor Networks (limited energy, low reliability, high sensitivity, etc.) on the other hand, several research works have addressed the security issue within WMSNs [6]. In fact, the emerging wide use of Wireless Multimedia Sensor Networks (WMSNs) applications, have stressed the development of securing techniques focusing either on secure multimedia data aggregation, communication protocol, privacy, quality of service, etc.

In general, we distinguish two main categories of approaches to secure images: (a) spatial-domain approaches in which the encryption process is applied at the pixel level in order to change its value and its position (i.e., substitution and permutation) in order to destroy the correlation between pixels. This process is based on different mechanisms such as DES [7], AES [8], asymmetric-key standards (where two keys are used (one public key that is published and used to encrypt the message, and another private key that is kept secret and used by the intelligible receiver to decrypt the message) as in RSA [9], wave transmission [10], elliptic curve [11], chaotic systems [12,13]. (b) frequency-domain approaches where the encryption process try to change the image data in the frequency domain based on some security key coefficients $[14,15]$.

Another interesting paradigm was introduced by integrating the hash functions with the image encryption field to provide message authentication and integrity [16], as well as to ensure data confidentiality. A hash-key-based encryption scheme has been discussed in [17], where the salsa20 hash function [18] is used to generate a dynamic secret key. Then, the produced key is correlated later with the plain-text image. In this paper, we named this work SALSA, and we refer to it as an example about a totally image encryption scheme that achieves data confidentiality without compression.

Moreover, we investigated other techniques that aim to reduce the amount of transmitted data whilst preserving its confidentiality, and several compression-encryption techniques have been proposed. In this context, data is first compressed using a known compression standard such as JPEG, JPEG 2000 and JPSec [19]. Then, bytes of the compressed code-stream follow the encryption process. A selective encryption scheme has been presented in [20]. In this approach, a piece-wise linear chaotic map (PWLCM) is used to generate the pseudo-random sequences. JPEG-2000 compression standard is applied to the original image. Then, the encryption process is performed on each 2-byte block of the packet body using a bitwise exclusive (OR) and some cyclic rotation operations in order to achieve a high randomness. Unfortunately, applying the encryption in all bytes of packet data (i.e., a huge amount of data to be encrypted) reduces the efficiency of this algorithm in terms of bandwidth and execution time. In this paper, we called this approach Compression-Encryption and we refer to it as an example about a combined compression-encryption scheme.

Finally, the proposed approach in [3], rather than using standard compression techniques, it reduces the amount of transmitted data by applying a novel technique based on Voronoi tessellation. Then, an efficient encryption technique is applied to ensure data confidentiality. We named this approach as Voronoi, and we refer to it as an example about a novel low complex compression-encryption technique. 


\section{Preliminaries}

In this section, we review the general scheme of Voronoi-based approach [3] to encode and decode sent images between two communicating nodes. We mainly highlight its components and how it works. We also review network techniques dedicated to error control during transmissions. Table 1 summarizes the main notations used in this paper.

Table 1: Table of Notations

\begin{tabular}{|c|l|}
\hline Notation & Definition \\
\hline$M K$ & Master Key \\
$D K_{v}$ & Dynamic Key \\
$I V$ & Initialization vector \\
$N$ & Number of Voronoi sites \\
$C V$ & Color Vector \\
$L$ & Number of lines \\
$C$ & Number of columns \\
$P$ & Number of plane (in gray-scale $\mathrm{P}=1)$ \\
$C P$ & Consecutive Perturbation \\
$G P$ & Gaussian Perturbation \\
$U P$ & Uniform Perturbation \\
$3 C$ & Closest Color Cell Filtering \\
$A C C$ & Average Color Cell Filtering \\
$\alpha$ & Number of retransmissions \\
\hline
\end{tabular}

\subsection{Voronoi Approach}

Voronoi approach consists of three steps: (1) Dynamic key Generation, (2) Encryption by the transmitter and (3) Decryption at the receiver side. Figure 1 represents the encryption, transmission and decryption mechanisms of this approach. Below, we provide a brief description of these steps.

\subsubsection{Dynamic key Generation}

Due to the weakness of using one static key (i.e., since it remains unchangeable for the whole network lifetime), the proposed approach makes use of a dynamic key (i.e., that is changed for every input image). The generation of dynamic key is performed using the following steps:

1. First, a single master key $M K$ is shared securely between the source and the destination nodes before establishing any communication. In [21], many propositions for the key exchange in constrained devices, especially in Wireless Sensor Networks (WSNs) have been discussed. One of them can be greatly integrated to efficiently respond to this issue.

2. Second, the used master key $M K$ is XOred with an initialization vector $I V$ of size 128-bits (i.e., IV must be unpredictable and nonce) to perform an output $K$.

3. After that, $K$ is concatenated with the counter $v$ (i.e., that is incremented for every new input image) and $I D_{A}$ ( i.e., the identity of the transmitter), then hashed using the hash function SHA-512 to produce an output $D K_{v}$. 


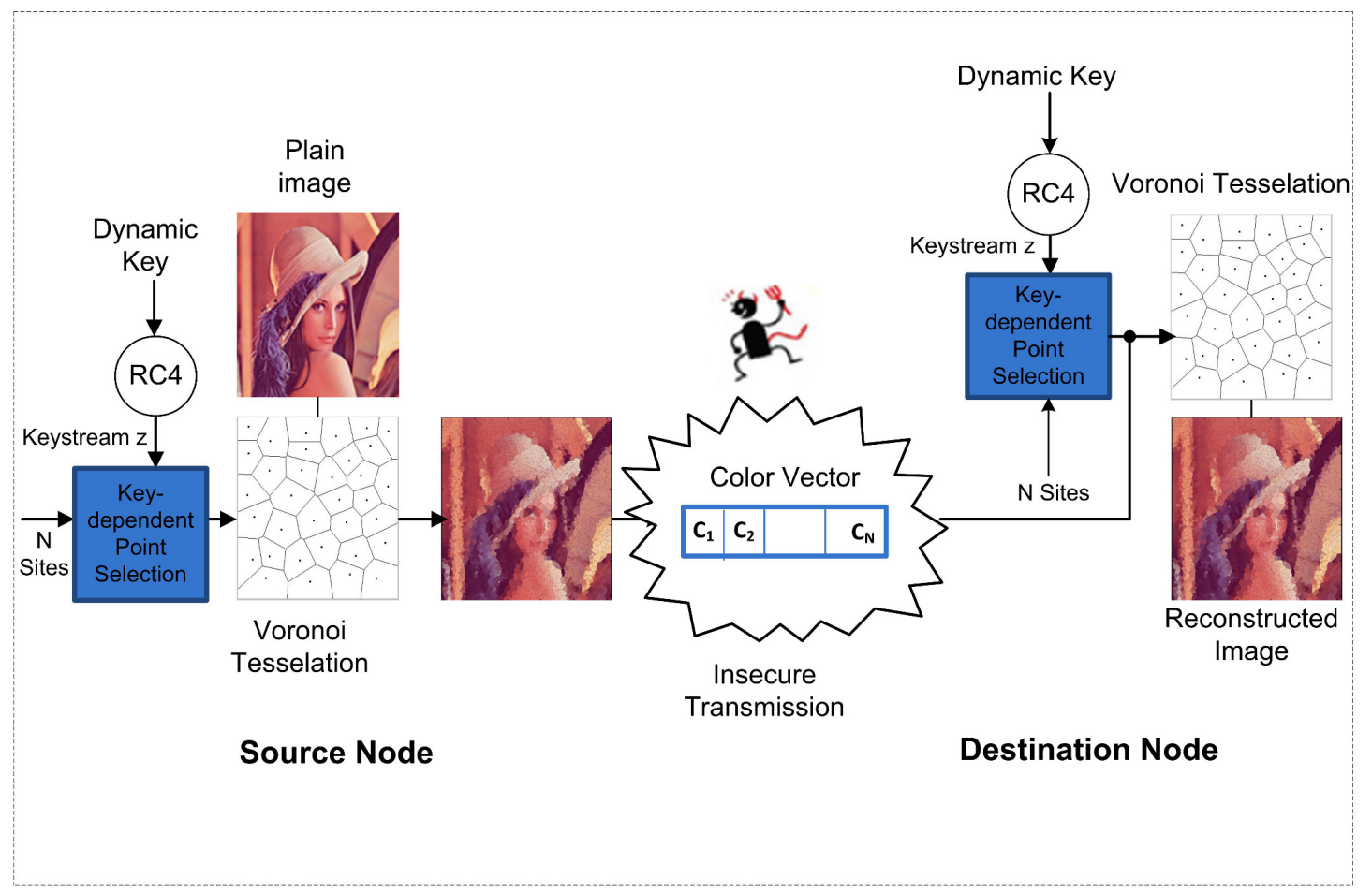

Figure 1: Functional diagram of source and sink nodes in the Dynamic proposed Approach.

4. After the generation of the dynamic key $D K_{v}$, a linear transformation is performed to generate the new coordinates $\left[\left(x_{1}^{\prime}, y_{1}^{\prime}\right),\left(x_{2}^{\prime}, y_{2}^{\prime}\right),\left(x_{3}^{\prime}, y_{3}^{\prime}\right), \ldots,\left(x_{N}^{\prime}, y_{N}^{\prime}\right)\right]$ of the $N$ Voronoi sites with coordinates $\left[\left(x_{1}, y_{1}\right)\right.$, $\left.\left(x_{2}, y_{2}\right),\left(x_{3}, y_{3}\right), \ldots .,\left(x_{N}, y_{N}\right)\right]$. The control parameters of this transformation, which are computed from the dynamic key. After that, these new coordinates are used to construct the Voronoi tessellation. The process of transformation is realized as below:

$$
\left[\begin{array}{l}
x^{\prime} \\
y^{\prime}
\end{array}\right]=T \times\left[\begin{array}{l}
x \\
y
\end{array}\right]=\left[\begin{array}{cc}
a & a+1 \\
a-1 & a
\end{array}\right]+\left[\begin{array}{l}
r x \\
r y
\end{array}\right] \bmod \left[\begin{array}{l}
L \\
C
\end{array}\right]
$$

Where $(x, y)$ are the initial coordinates of one point, selected from the sequence of $N$ sites, and $\left(x^{\prime}, y^{\prime}\right)$ represent its corresponding new coordinates after applying the required transformation. $a, r_{x}$ and $r_{y}$ are the control parameters of this transformation that are computed directly from the dynamic key $D K_{v} . L$ and $C$ represent the size of lines and columns of the input image, respectively.

This transformation is iterated 4 times, to avoid the unique permutation sequence generation, where the same sequence is generated twice. Each iteration necessitates the use of three different control parameters $a, r_{x}, r_{y}$. Hence, as a result, 12 control parameters are needed to be used to perform the transformation of each point $(x, y)$. In this case, the dynamic key $D K_{v}$ of size 128 bits is divided into 12 components each of size of 10 bits, and each 3 components are used as control parameters $\left(a, r_{x}, r_{y}\right)$ in each iteration.

\subsubsection{Encryption at transmitter side}

Based on the generated $\mathrm{N}$ sites, the Voronoi tessellation is applied on the input image. After that, the color of each voronoi cell is computed, and represented by the mean of all pixels belonging to this cell. Each computed color is stored sequentially in a vector called Color Vector (CV). After computing the colors of all Voronoi cells, CV is ready for transmission to the receiver. Let's note that, instead of transmitting the 
whole image, only CV components need to be transmitted to the receiver. Thus, it significantly reduces the transmission bandwidth.

\subsubsection{Decryption at the receiver side}

Upon receiving the color vector $\mathrm{CV}$ from the transmitter side, the receiver tries to reconstruct the original image as follows: First, as previously mentioned, $M K$ is shared secretly between both intelligible parties, hence a similar procedure is applied to generate the dynamic key $D K_{v}$ from $M K$. Additionally, the same steps are applied to the dynamic key to generate the coordinates $\left(x^{\prime}, y^{\prime}\right)$ of all $\mathrm{N}$ points (i.e., the dynamic linear transformation is applied on each $(x, y)$ coordinates). After that, based on these $N$ points, the receiver builds the Voronoi diagram. Finally, the components of the received $C V$ are added sequentially to perform the color of each Voronoi cell. Indeed, the reconstruction of the original image is accomplished.

\subsection{Transmission Errors Control}

Data link layer (DLL) is the second layer in the OSI (Open System Interconnection) reference model Stack [22]. This layer provides a reliable and efficient data transfer over two connected nodes. In other words, it is responsible for handling the communication over the physical link between network entities, and might provide functional means to detect and correct errors that may occur on physical layer [23]. Data link layer is divided into two sub-layers:

- Media Access Control (MAC): controls the accessing of computers in the network gain to the data, and the manner of obtaining the permission to transmit this amount of data.

- Logical Link Control (LLC): controls the flow of messages between stations, checks and corrects errors.

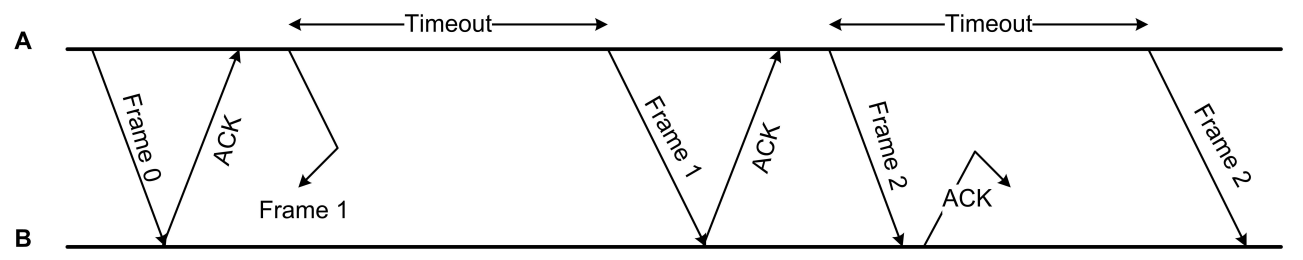

Figure 2: The ARQ communication protocol

The main goal of data link layer is to make the physical layer appears as error-free to the higher layers, in addition of ensuing data transparency (the receiver gets the data in the same form that was transmitted by the sender). Frame is the fundamental basic unit used to control the flow of messages. Each frame is a sequence of bits or bytes (i.e., most basic units of information in computing and communication). It consists of four parameters: (a) type, which indicates if the frame is a data frame or a control frame, (b) sequence number, which represents the number of the frame, (c) acknowledgment, which indicates the successful receipt of the frame, and (d) info, which contains the data to be sent.

Two types of error checks are added to the frame in order to identify the erroneous frames: (1) the first type is the CRC check [24], (2) and the second type is the checksum [25]. Both allow the receiver to check whether or not the received frame is correct. Indeed, upon receiving a frame from the physical layer, the receiver tries to recalculate the checksum and compare its value with that contained in the frame. If both values are equal, the frame is accepted. Otherwise, the frame is rejected.

The most widely-used protocol for error control in wireless sensor networks is the Automatic Repeat reQuest $A R Q$ [4]. Under this protocol, the transmitter first constructs its data frame by setting up a sequence 
number for each data frame. Then, it sends it to the physical layer and starts a timer. The transmitter keeps transmitting the same frame until it gets a positive ACK, which carries the sequence number of the next expected frame. When the receiver gets the data frame, it checks whether the sequence number is the expected sequence number; if not, it discards the frame. If the receiver finds that this is the expected frame, it detects whether the received frame contains an error, by calculating the checksum. If an error is occurred, the frame is discarded, and a retransmission of the same frame is performed. Also, when the transmitter does not receive an ACK within the expected time, it resends the frame. Figure 2 illustrates two retransmission scenarios: (1) the first is when the frame is lost, while (2) the second occurs when the ACK is lost. In this Figure, the following sequence of events have occurred:

1. Node A transmits a frame 0 and waits for an ACK frame from the receiver node B.

2. Frame 0 arrives to the receiver $B$ without error. So, B transmits an ACK frame.

3. The ACK of B is received without error, so A knows that frame 0 has been received correctly.

4. Node A now proceeds to transmit frame 1 and resets the timer.

5. Frame 1 undergoes error in transmission. It is possible that the node B receives frame 1 and detects error by the CRC check. On the other hand, it is also possible that frame 1 has badly garbled. In both cases, Node B does not take any action.

6. The timeout period is expired, and frame 1 is retransmitted.

7. Frame 1 arrives at the receiver B without error. So, B transmits an ACK frame.

8. The ACK of B is received without error, so A knows that frame 1 has been received correctly.

9. Node A now proceeds to transmit frame 2 and resets the timer.

10. Frame 2 arrives to the receiver $B$, which in turn sends an ACK frame to A.

11. Node A does not receive the ACK frame. When the timeout period is expired, A resends frame 2 to the receiver $\mathrm{B}$.

Indeed, there are three cases in which a retransmission of the frame is required: (a) loss of the frame, (b) corruption of the frame, and (c) the loss of ACK. Hence, the major drawback of this protocol is the additional cost incurred from retransmissions [4], especially in noisy environments.

In our proposal scheme, as only the color vector $\mathrm{CV}$ is transmitted via the noisy channel to the receiver, we propose a variant protocol derived from ARQ scheme which allows the transmission errors to be handled in the upper layer (i.e., application layer). By doing so, data retransmissions can be saved, thus leading to an important network performance improvement, mainly in term of energy conservation. We provide details about this protocol in the next section.

\section{The Proposed Errors Resilient Scheme}

The main feature of our proposed resumed by giving the application layer the responsibility of handling communication errors, instead of the lower layer (i.e. data link layer). Thus, it is achieved by investigating a recovery mechanism that is able to correct the corrupted data in order to enhance the visual quality. Indeed, this method reduces the communication overhead by eliminating the retransmissions overhead induced either by communication failures or by active attacks.

In this context, the proposed scheme consists of two main components: (a) the first component works at the physical layer, and is defined by a communication protocol that can detect corrupted data and report them to the upper layer, while (b) the second component works on the application layer and is composed of a recovery mechanism capable, to a certain extent, of reconstructing the image from the corrupted received data. Details about these two components are discussed in the next few sections. 


\subsection{The Proposed Communication Protocol}

In order to control errors during the transmission of color vector CV over an unreliable channel, especially when dealing with constrained devices (i.e., wireless sensor networks WSNs), we propose a variant communication protocol, inspired from the ARQ scheme. Due to the intrinsic features of the proposed Voronoi algorithm, the proposed communication protocol is based on dividing the components of Color Vector (CV), which are already sent by the transmitter, into several frames, each of which contains one component of the color vector (i.e., each color is transmitted via one frame). All the components of the color vector are labeled sequentially and sent in this order. This protocol consists of the following steps:

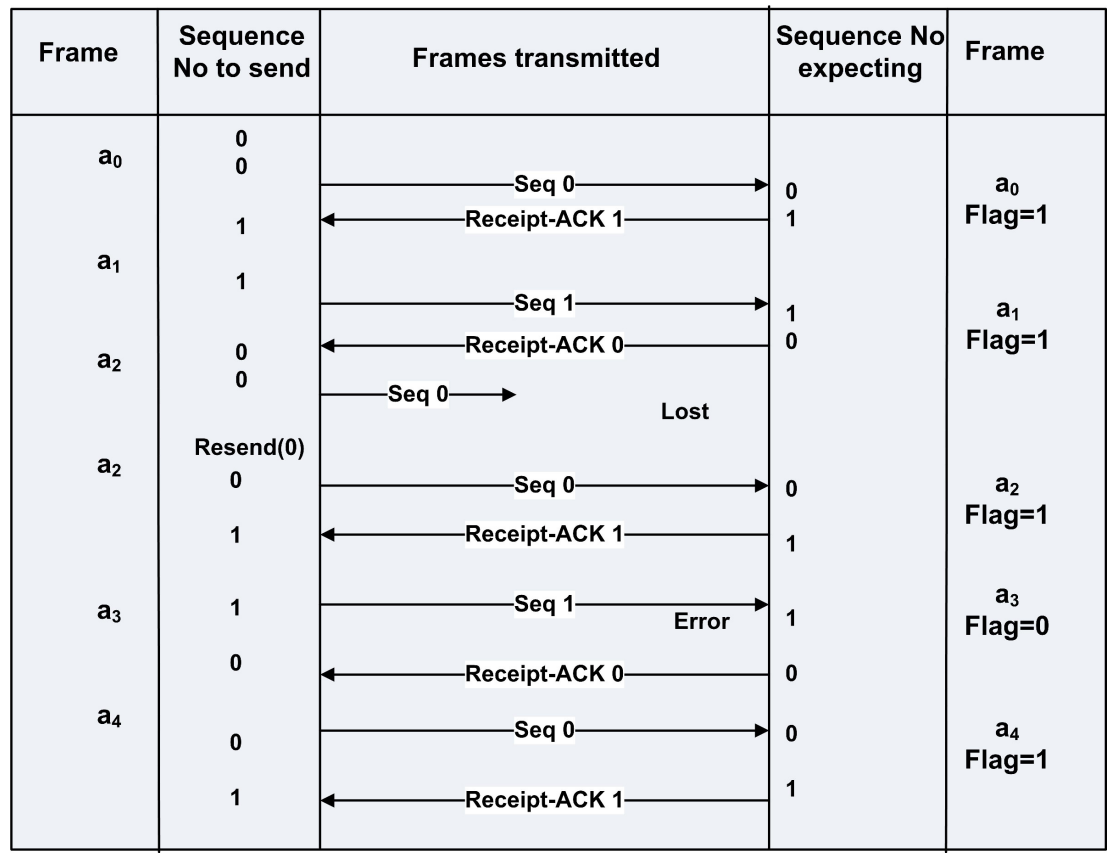

Figure 3: Flow of data between the sender and the receiver using the proposed protocol

1. Similarly to ARQ scheme, the transmitter sends its data frame to the physical layer.

2. At the physical layer, and before sending the frame to the receiver, a checksum sequence is added to each received frame.

3. Upon receiving a frame, the receiver acknowledges the reception of the frame by sending a $R e$ ceipt $A C K$ to the sender.

4. The receiver verifies whether or not the frame is correct (i.e., corrupted) by checking its checksum, in the same way as it is done in ARQ.

5. For each received frame, a flag named Error Flag (EF) is added, to indicate if the received frame is correct or if it has been corrupted during transmission.

6. Finally, a re-ordering of all received frames with their corresponding EF, according to their initial order is done, before transmitting them to the upper layer, where corrupted frames will be handled.

The overall protocol diagram is illustrated in Figure 3. We note that, within this protocol, frame retransmission occurs only when either a transmission of a frame or a Receipt $A C K$ is lost. In both cases, a time_out is detected at the sender side, which triggers a retransmission of the current frame. However, the erroneous frames are passed to the higher layer, where errors are corrected using different recovery mechanisms. 


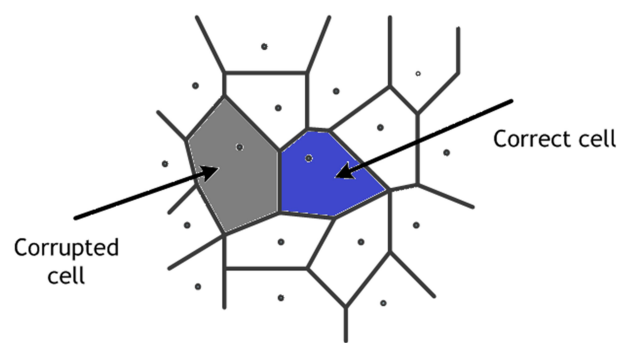

(a)

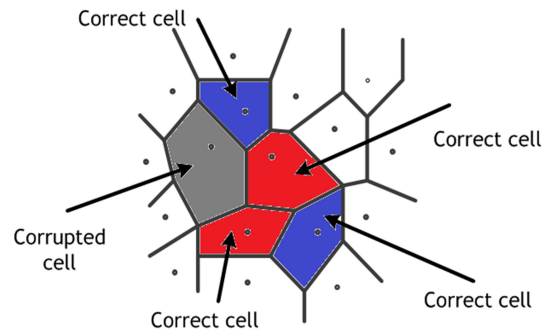

(c)

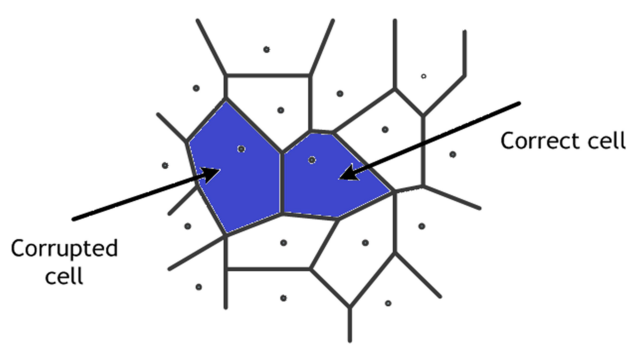

(b)

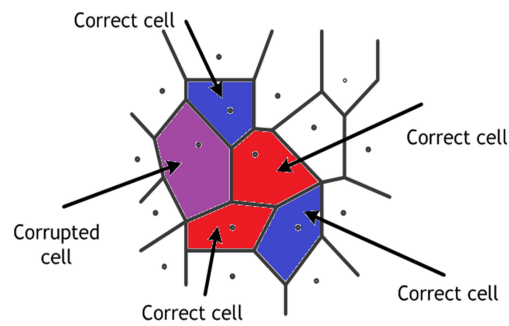

(d)

Figure 4: (a),(b) Closest Color Cell (3C) recovery mechanism. (c),(d) Average Color Cell recovery mechanism.

\subsection{The Proposed Recovery Filtering}

Upon receiving the color vector at the destination side, the receiver applies first the decryption process as explained in Sub-Section 3.1. After performing the Voronoi tessellation, the receiver tries to color this tessellation in order to reconstruct the original sent image. This is achieved, by filling the components of the color vector sequentially in the Voronoi tessellation. Unfortunately, according to the used communication protocol, some components of the received color vector have been corrupted during the transmission and do not necessarily correspond to the correct color. Using these corrupted values may lead, to a certain extent, to a degradation in the perceived quality of the reconstructed image. For this reason, two recovery filtering mechanisms have been proposed: (1) The Closest Color Cell (3C), and (2) the Average Color Cell (ACC).

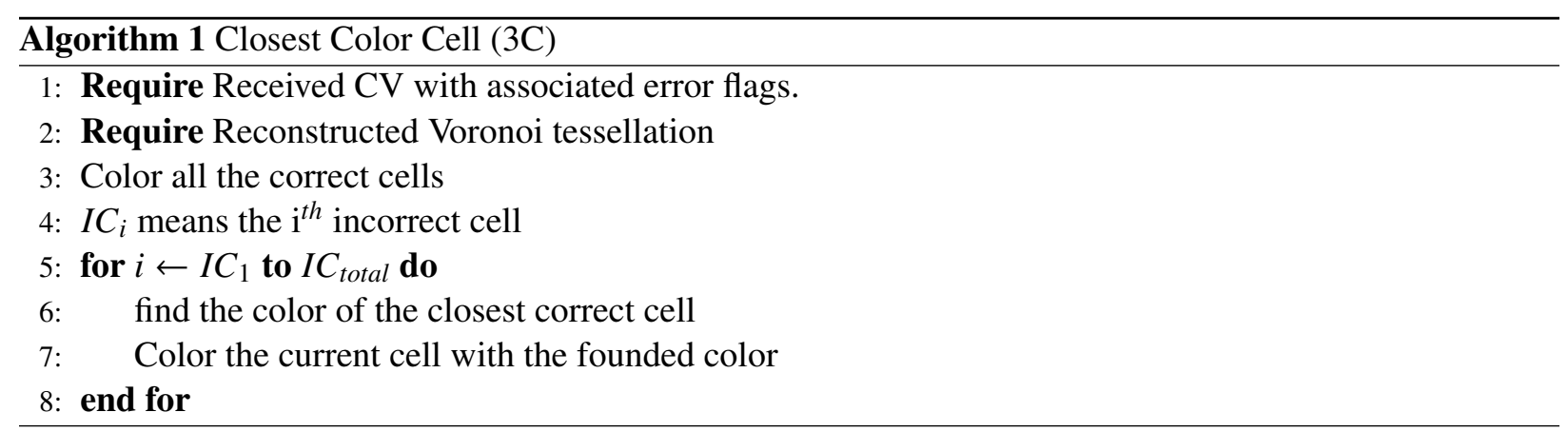

\subsubsection{Closest Correct Cell (3C)}

The first recovery filtering mechanism is called Closest Correct Cell (3C: triple C). This filter exploits the intrinsic characteristics of the used Voronoi tessellation, since the Voronoi diagram is nothing else than 
a spatial partition of the original image. Indeed, knowing the corrupted components of the color vector allows the corresponding Voronoi cell to be identified. From that, the filtering mechanism tries to exploit the intrinsic spatial redundancy already present in the original image, by extending the color of the closest correct cell to the corrupted cell, as illustrated in Figure 4-(a),(b). In addition, Algorithm 1 represents the corresponding pseudo-code of the proposed recovery filtering mechanism.

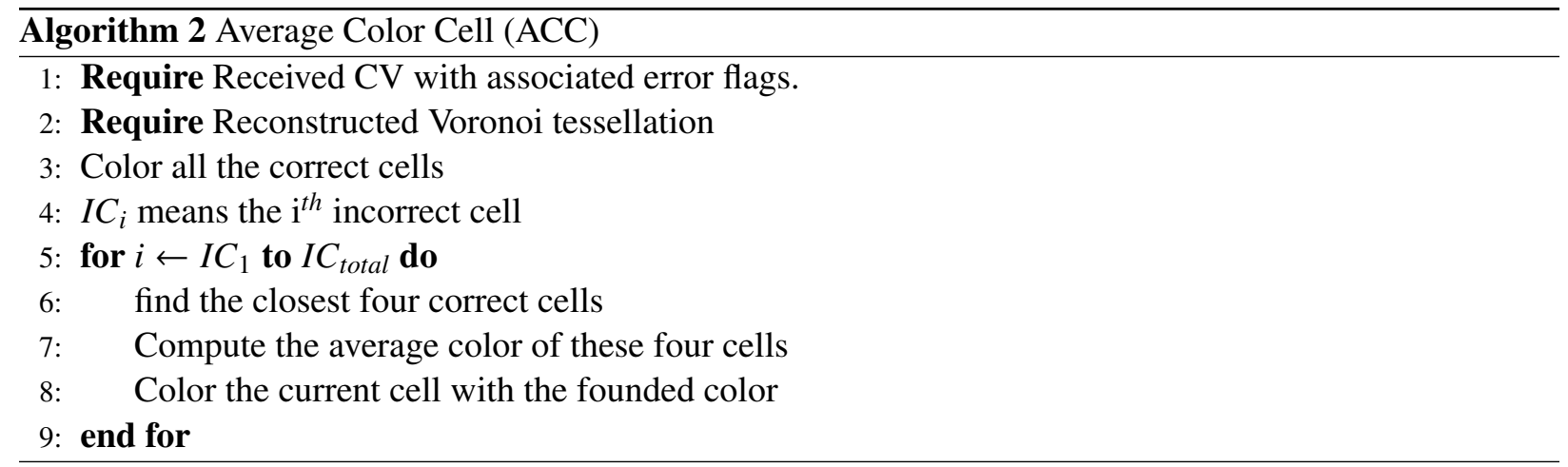

\subsubsection{Average Color Cell (ACC)}

In the before explained recovery filtering, the receiver tries to color each corrupted cell with the color of the closest corrected cell. This is done to increase the accuracy of the correction mechanism, especially in the case when the closest correct cell has a color that is in reality far away from the original color value. For example, we could suppose that in the Lena image, we attempt to correct the color of one corrupted cell located in the hat seen in the Lena image. Then, we find that the closest correct cell is located in the hair of the Lena image. If we used the first filtering technique, the corrupted cell will be replaced by a Black color instead of Beige color, which corresponds to the original cell color. For this reason, another recovery filtering is proposed and studied. This filter is based on coloring the corrupted cell with the average color of four neighboring correct cells. In this context, for each corrupted cell, first we find the four closest neighboring correct cells. Then, the mean color of the corresponding cells is computed and used to fill the color of this erroneous cell. Figure 4-(c),(d) illustrates the mechanism of this recovery filtering, and Algorithm 2 explains the procedure used in this filtering.

\section{Performance Evaluation}

In this section, we evaluate the performance of our proposed error-resilient scheme in terms of reducing data retransmissions due to the occurrence of errors, whilst maintaining unchanged the perceived visual quality of the reconstructed images. To this end, we have conducted several series of simulations using MATLAB R2013b simulator [26] to measure the error resilience under different types of perturbations. For the visual image quality measurements, we have used the two well-known metrics: Peak-Signal to Noise Ratio (PSNR) [27] and Structural Similarity Index (SSIM) [28] to measure the quality of reconstructed images. The first one is derived from the Mean-Squared Error (MSE). Although, it is known as the most commonly-used metric for comparing images, it suffers a slight limitation in applications where the processed images are meant to be viewed by humans [29]. For this reason, the second metric (i.e., SSIM) has specifically been developed to be more compliant with the Human Visual System (HVS). Additionally, the three standard images: Lena, Peppers and Baboon, each of size $512 \times 512 \times 3$ are used to apply the series of experiments. 
For comparison purposes, we have implemented, in addition to our approach, another two approaches: SALSA, which has been proposed in [17] and explained in Section 2. This approach is used as an example of image-encryption only schemes. The second approach is Compression-Encryption which has been presented in [20] and explained in Section 2. We take this approach as an example about the traditional joint compression-encryption schemes. We note that this approach is very close to our proposed Voronoi scheme, since it fulfills data reduction while preserving data confidentiality. The difference is that in our scheme, we have reduced the amount of transmitted data using Voronoi tessellation, which is far away from the traditional compression techniques.

\subsection{Settings}

Before presenting the performance evaluation results, we first present the considered scenarios in which errors are introduced (i.e., types of perturbations). We also present the image quality parameters that we have fixed through experiments.

\subsubsection{Types of Perturbations}

We have considered several scenarios in which sent data is perturbed during transmission either by an attacker or due to errors. In each scenario, the content of the transmitted data is altered: (a) the color vector in our approach, (b) the cipher image in SALSA, and (c) the encrypted packet in CompressionEncryption. The amount of altered data has been varied and studied according to three known types of noise perturbations: Consecutive, Uniform and Gaussian [30].

- Consecutive Perturbation $(C P)$ : In this type of perturbation, a malicious attacker tries to hide the image content by modifying some components of the transmitted data with another value (i.e., another color), in a contiguous manner. This could happen from one-shot attack or one perturbation during which a portion of the sent data is corrupted.

- Uniform Perturbation $(U P)$ : In this type of perturbation, the noise is introduced by an attacker through a quantization of some components of the transmitted data, using different quantization levels. The resultant gray values (0-255) of the noise are uniformly distributed across the whole data. The probability density function (PDF) of the uniform distribution is given by:

$$
f(x)= \begin{cases}\frac{1}{b-a}, & \text { if } a \leq x \leq b \\ 0, & \text { otherwise }\end{cases}
$$

- Gaussian Perturbation (GP): In this type of perturbation, the produced noise in some components of the transmitted data is an additive in nature, following a Gaussian distribution. Hence, the noisy pixel value is the sum of the true pixel value and a random Gaussian distributed noise. The related PDF is expressed by the following equation:

$$
f(x)=\frac{1}{\sqrt{2 \pi \sigma}} e^{-\frac{(x-\mu)^{2}}{2 \sigma^{2}}}
$$

where $x$ is the gray level, $\mu$ is the mean of the function, and $\sigma$ is the standard variation of the noise. In our implementation, we use a Gaussian distribution with $\mu=0$ and $\sigma=0.5$.

Upon receiving of noisy data, the receiver tries to reconstruct the image. We then measured the difference between the sent image and the reconstructed one for the three different studied approaches. 


\subsubsection{Image Quality Parameters}

One of the intreresting feature of Voronoi approach is its high flexibility in the sense that the number of Voronoi sites (e.g., $N$ ) is controlled by the transmitter and hence it can adapt the desired visual quality. In other words, depending on the application requirements, the transmitter decides to decrease/increase $N$. For example, if the transmitter goal is to preserve a high image quality, $N$ value must be increased. On the other hand, if the interest is to preserve the network resources, a lower value of $N$ must be chosen. In [3], the impact of this parameter on the image quality has been evaluated using extensive experiments, and for different types of images. Results have proved that for a value of $N$ equals to $1 \%$, a good SSIM index has been attained (i.e., SSIM=0.7 for an image of size $512 \times 512 \times 3$ ). Additionally in this paper, we have studied two additional parameters:

\section{The image size}

The size of the image is expressed by $L \times C \times P$, where $L$ is the number of rows, $C$ is the number of columns and $P$ is the number of plane ( $P=1$ in gray scale image). Our proposed approach is based on taking $N \%$ of the total amount of pixels existing in an image. Hence, with the variation of image size, the value of $N$ is changed, and the image quality is affected as well.

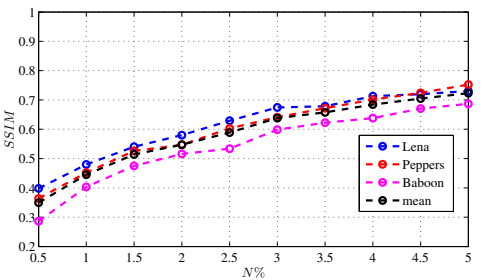

(a)

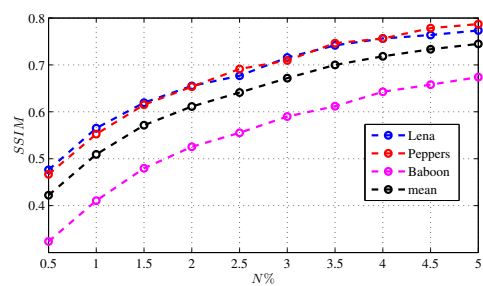

(b)

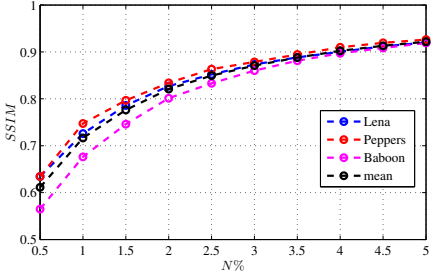

(c)

Figure 5: (a),(b),(c)-The variation of SSIM index using different sizes of images: $128 \times 128 \times 3,256 \times 256 \times 3$ and $512 \times 512 \times 3$ respectively and with different $N \%$.

To evaluate this metric, we have studied the variation of SSIM index for three standard color images: Lena, Pepper and Baboon with different image sizes $(128 \times 128 \times 3,256 \times 256 \times 3$ and $512 \times 512 \times 3)$ and with $N$ value that changes from $N=0.5 \%$ to $N=5 \%$. The results for all image sizes are illustrated in Figure 5-(a),(b),(c) respectively. From the results, we can deduce that: (a) for an image size of $128 \times 128 \times 3$, the $N$ value that results on a good SSIM index ( $\geq 0.7)$ is $4 \%$; (b) For an image size of $256 \times 256 \times 3, N=3 \%$ is sufficient; (c) also, for an image size of $512 \times 512 \times 3, N=1 \%$ is sufficient. In this work, we deal with images of size $512 \times 512 \times 3$, for this reason, we fix $N$ to $1 \%$.

\section{The pixels ditribution among Voronoi cells}

The distribution of pixels among Voronoi cells depends directly on the image size, as well as on the chosen $N \%$ value. For a fixed $N$ value $=1 \%$ and with a small image size $(128 \times 128 \times 3)$, pixels are distributed among all Voronoi cells in a way that each Voronoi cell contains a little number of pixels, which differ from one cell to another, but remains approximately low in all Voronoi cells. On the other side, with the increase of the image size $(256 \times 256 \times 3)$, the distribution of pixels becomes less condensate, where some Voronoi cells contain a large number of pixels while a small number of pixels are founded in others. This analysis is proved with the results shown in Figure 6, where histograms corresponding to the distribution of pixels among voronoi cells for Lena image with different sizes: $128 \times 128 \times 3,256 \times 256 \times 3$ and $512 \times 512 \times 3$ and with $N=1 \%$ are illustrated. 


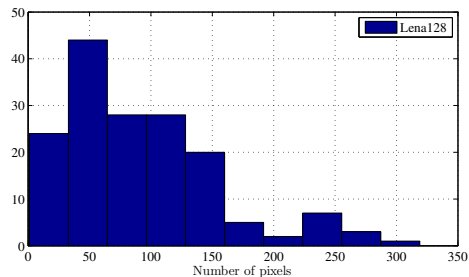

(a)

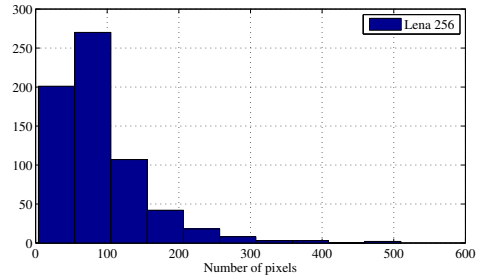

(b)

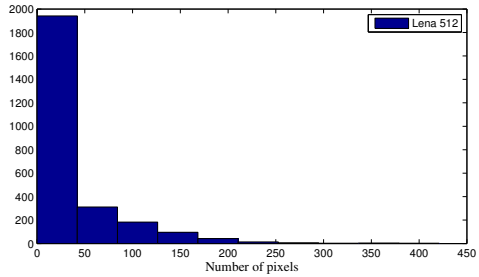

(c)

Figure 6: (a),(b),(c)-The distribution of pixels between all Voronoi cells for Lena image with size: $128 \times 128 \times 3,256 \times 256 \times 3$ and $512 \times 512 \times 3$ respectively and $N=1 \%$.

\subsection{Results}

\subsubsection{Error Resilience without filtering}

In the first step, we wanted to evaluate the level of error resilience of each approach, without applying any recovery filtering techniques. To this end, we have conducted a series of experiments using the three well-known standard images: Lena, Peppers and Baboon $($ size $=512 \times 512 \times 3)$. We have varied the percentage of perturbations from $1 \%$ to $20 \%$, using the three types of perturbations, on each sent image. Then, we have measured the two metrics PSNR and SSIM between the transmitted image and the finally received corrupted image. We have plotted the curves of the mean values of PSNR and SSIM indexes for the three images under each perturbation type and for each approach. Results are shown in Figures 7, 8 and 9.

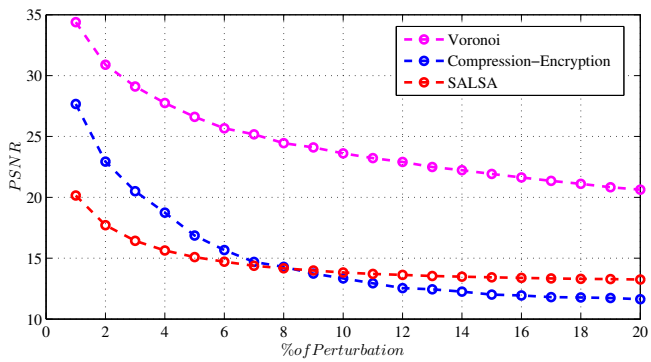

(a)

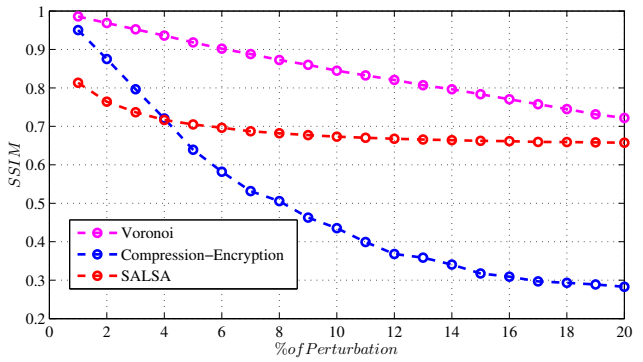

(b)

Figure 7: The variation of PSNR and SSIM indexes under Consecutive perturbation.

The first conclusion that we can extract from these results is that the quality of the reconstructed images decreases with the increase of noise, independently of its type. Also, the impact of noise varies with each type, and has different intensities between the different studied approaches.

We begin first by analyzing the results of perturbations in SALSA approach. As illustrated the figures, results for PSNR as well as for SSIM values demonstrate that the impact of UP is the most important, especially for the SSIM index (i.e., for UP perturbation, the mean of SSIM index decreases from 0.8671 to 0.2952). This is because in this image encryption approach, the goal is to satisfy the avalanche criterion, where any little change on the ciphertext must produce more than $50 \%$ of change in the decrypted image. Indeed, SALSA approach is very sensitive to any little change that may occur in its key, as well as in the ciphertext pixels. Additionally, the uniform perturbation is based on uniformly distributing the noise over the whole encrypted image. So, the decryption of this image must support more degradation compared to CP or GP. 


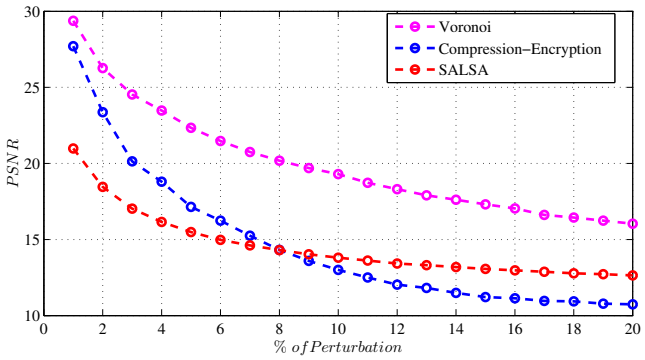

(a)

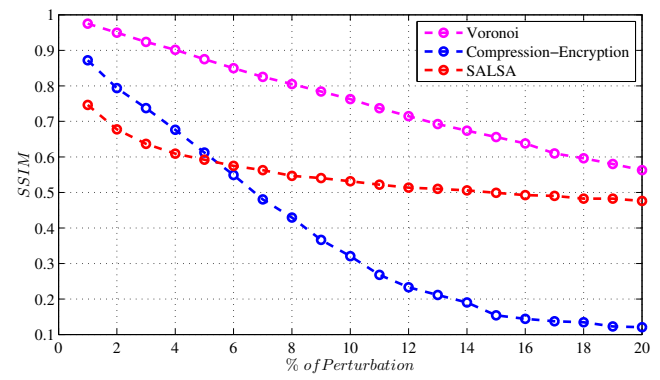

(b)

Figure 8: The variation of PSNR and SSIM indexes under Gaussian perturbation.

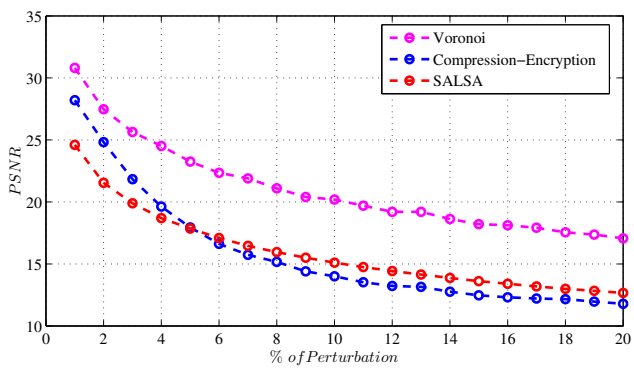

(a)

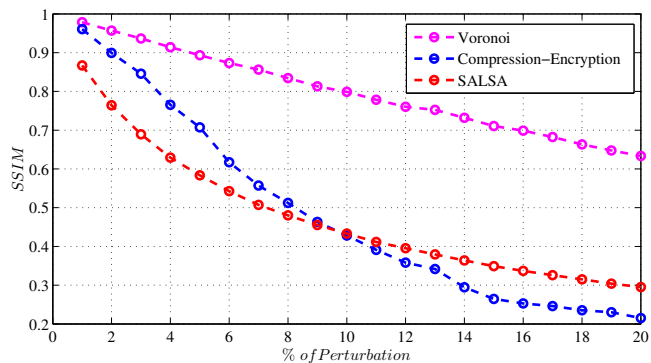

(b)

Figure 9: The variation of PSNR and SSIM indexes under Uniform perturbation.

In Compression-Encryption approach, errors are added to the encrypted code-stream, which is composed of packets of varying sizes. The effects of UP and GP are more important than CP, but in all perturbations, the image under this approach begins with less degradation compared to the SALSA approach. Then, after approximately $4 \%$ (for CP and GP), and $10 \%$ for UP, it becomes more distorted than the SALSA approach. Thus, it occurs because compression adds randomness to the system in order to facilitate the encryption task. Additionally, the tested Compression-Encryption scheme is based on XORing each two bytes in the packet body to follow the encryption process. Indeed, any error that affects one byte will be transmitted to the subsequent bytes. Furthermore, results of PSNR and SSIM values for Voronoi approach, illustrated in the figures, show that the impact of perturbation is more important in UP and GP compared to CP. The reason behind these results is due to the features achieved by this algorithm, which is based on breaking the spatial redundancy that exists in images. Hence, when the perturbations are distributed over the image, their impact is less pronounced when they are concentrated on one region, which reflects the obtained results.

Quantitative results also demonstrate the effectiveness of Voronoi approach over Compression-Encryption and SALSA approaches. Indeed, the PSNR of Voronoi approach attains a higher value under the different types of perturbations and for the three tested images, even with a higher percentage of errors (for 15\% of errors, the mean of PSNR in Voronoi equals to $21.9182 \mathrm{~dB}$ for CP, 17.3147 dB for GP and 18.2080 $\mathrm{dB}$ for UP). In contrast, for SALSA approach, a lower PSNR value is attained (for $15 \%$ of errors, mean PSNR value is equal to: $13.4318 \mathrm{~dB}$ for CP, $12.1376 \mathrm{~dB}$ for GP and $13.4019 \mathrm{~dB}$ for UP). The lowest PSNR values are attained by Compression-Encryption approach (for $15 \%$ of errors, mean PSNR value is equal to: 
$12.0137 \mathrm{~dB}$ for $\mathrm{CP}, 11.2201 \mathrm{~dB}$ for GP and $12.3087 \mathrm{~dB}$ for UP).

Similarly, for the three approaches, we have measured the SSIM index. In Compression-Encryption approach, SSIM index attains a lower value beginning by 0.8739 for $1 \%$ of errors and decreasing to attain a very low value (for 15\%, SSIM in Compression-Encryption is equal to: 0.2878 for CP, 0.1541 for GP and 0.2646 for UP). In SALSA approach, the effect of errors is less important than Compression-Encryption approach (after 5\% of errors in CP and GP and 10\% of errors in UP) and more important than Voronoi approach (for 15\%, SSIM is equal to: 0.6527 for CP, 0.4990 for GP and 0.3492 for UP). Finally, Voronoi approach is the most effective approach against perturbations, where the SSIM index remains high even with a high percentage of errors (for 15\%, SSIM in Voronoi is equal to: 0.7836 for CP, 0.6558 for GP and 0.7108 for UP), which means that the image conserves a relatively good visual quality. However, beyond $15 \%$ of perturbations, SSIM index decreases to attain lower value and the image degradation for Voronoi approach becomes highly noticeable.

From that, we can conclude that SALSA and Compression-Encryption approaches are less resilient to error propagation, compared to the Voronoi approach. In SALSA approach, the entire image is directly affected. Also, the aim of encryption under this approach is to achieve the avalanche effect. Indeed, any little change in the plaintext will lead to a huge degradation in the ciphertext. For Compression-Encryption approach, errors do not affect the entire image; instead, the packets of the code-stream are affected, but the use of compression makes the bytes more random, which facilitates the propagation of errors. Additionally, the tested approach is based on encrypting the packet body, byte by byte, and XORing each two adjacent bytes (i.e., as if using CBC mode), which makes the encryption of one byte block dependent on its adjacent byte block. Indeed, any error that occurs in one byte block will propagate to the subsequent byte blocks.

To illustrate the visual effect of $15 \%$ of errors on the considered images, Figures 10, 11 and 12 represent Lena, Peppers and Baboon images for SALSA, Compression-Encryption and Voronoi approaches within consecutive, Gaussian and Uniform perturbations respectively. From the figures corresponding to Compression-Encryption approach, we can remark that in the three images and for the three perturbation types, images are significantly degraded.

The better behavior of Voronoi approach, as attested by the obtained results, is due to its intrinsic construction: by generating random and independent sites, Voronoi approach somehow breaks the spatial adjacency between sites, which allows it to distribute the errors over the whole image, even when they are contiguous in the transmitted color vector. This unique feature makes this approach less sensitive to errors, in comparison to other approaches. Figures 10,11 and 12 show examples of images before and after introducing 15\% of noise in SALSA, Compression-Encryption and Voronoi approaches for CP, GP and UP perturbations, respectively.

\subsubsection{Error Resilience With Filtering Mechanism}

Previous results show the intrinsic immunity of our proposed Voronoi approach, to a certain extent (i.e., $15 \%$ of errors). After this percentage, image quality is significantly degraded. For this reason, we have proposed two alternative filtering mechanisms: (1) 3C filter and (2) ACC filter (introduced in Section 4), that try to go further by exploiting on one hand the construction features of this approach and on the other hand the spatial redundancy of images.

We implement the two proposed recovery filtering mechanisms: (1) the Closest Correct Cell (3C) which is based on the idea of propagating the color of the closest correct cell and (2) the Average Correct Cell (ACC), which is based on coloring the corrupted cell with the mean color of its four neighboring corrected cells. The mean values of PSNR as well as of SSIM index are computed for the three tested images, under CP, GP and UP perturbations (i.e., the percentage of errors changes from $1 \%$ to $100 \%$ ). Results are plotted in Figure 13. 

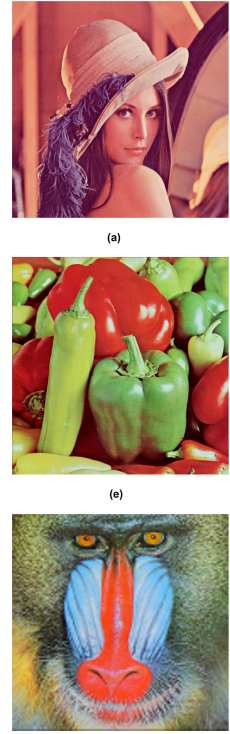
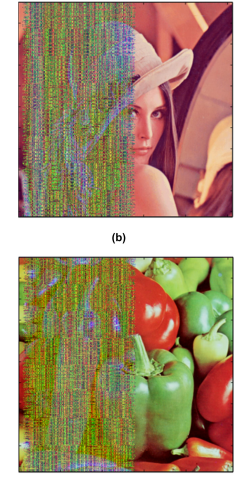

(f)

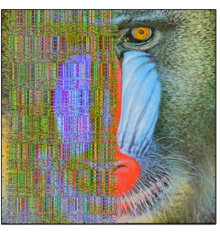

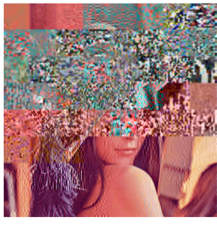

(c)
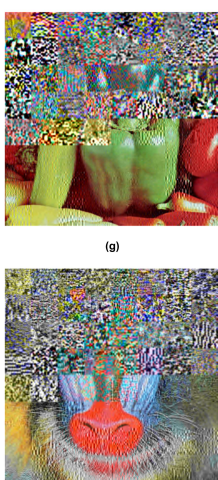

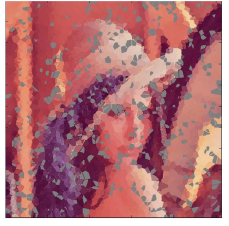

(d)
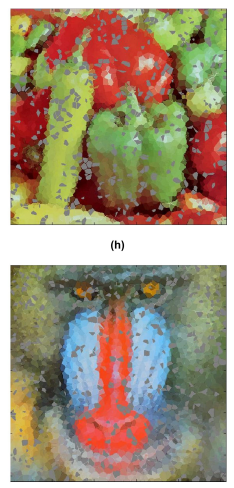

(CP)

Figure 10: CP: the effects of $15 \%$ of errors on the three standard images under CP perturbation, for SALSA, CompressionEncryption and Voronoi approaches, respectively.
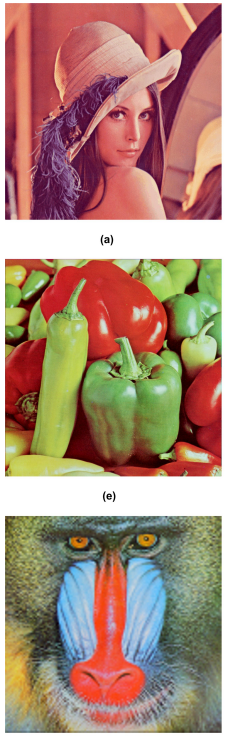

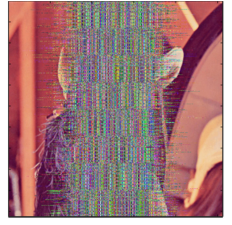

(b)

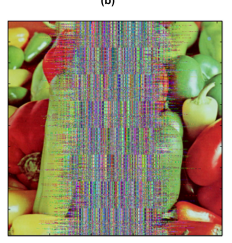

(f)

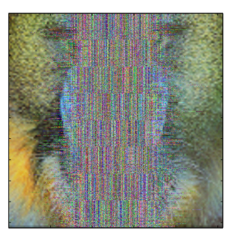

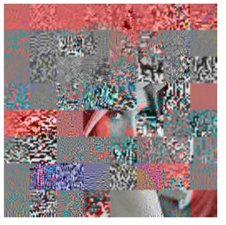

(c)
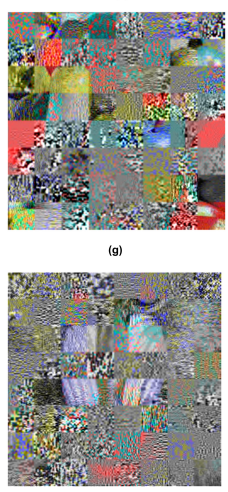

(GP)

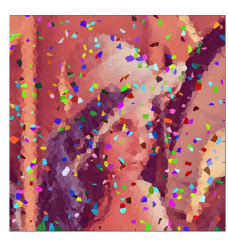

(d)
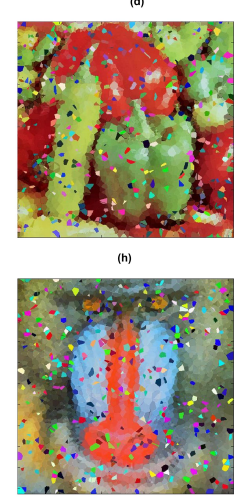

Figure 11: GP: the effects of $15 \%$ of errors on the three standard images under GP perturbation, for SALSA, CompressionEncryption and Voronoi approaches, respectively.

We begin with the $3 \mathrm{C}$ filter, where the mean values of SSIM and PSNR indexes attain a good value for a percentage of errors up to $25 \%$ for CP (at 25\%, SSIM=0.7108, and PSNR=18.7723). For GP and UP perturbations, SSIM and PSNR indexes remain good, until a percentage of errors equals to $20 \%$ (for GP, SSIM=0.6962 and PSNR=16.3842, while for UP, SSIM=0.7181 and PSNR=17.2552). After these percentages, both indexes attain lower value and the image is highly degraded with the increase of errors. Indeed, the $3 \mathrm{C}$ filter succeeds in improving the percentage of errors for the tested color image by an amount 

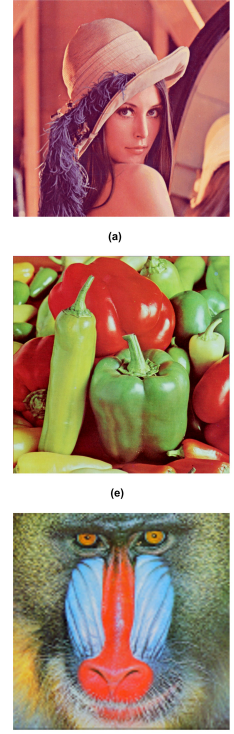

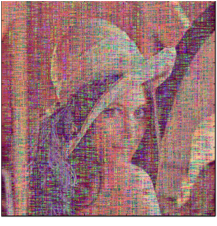

(b)
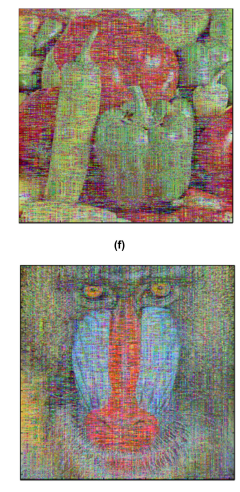
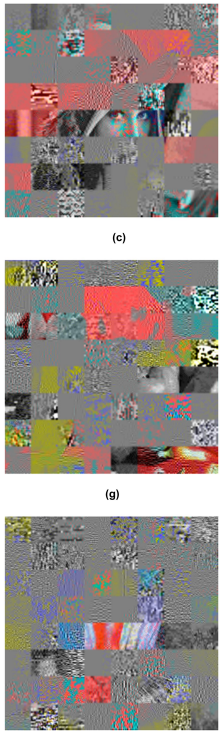

(k)

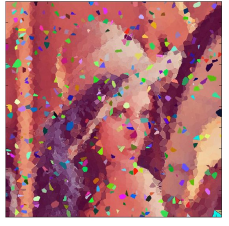

(d)
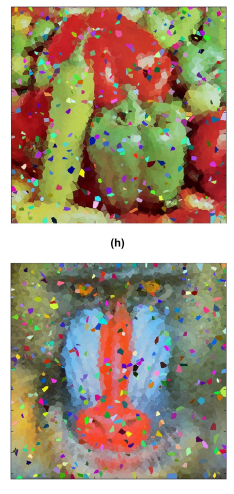

Figure 12: UP: the effects of $15 \%$ of errors on the three standard images under UP perturbation, for SALSA, CompressionEncryption and Voronoi approaches, respecitvely.

approximately equal to $5 \%$, passing from $15 \%$ to $20 \%$.

On the other side, the ACC filter significantly enhances the image quality. SSIM and PSNR indexes preserve a higher value with a high percentage of errors: At 90\%, for CP: SSIM $=0.7228$, PSNR=21.2159; For GP: SSIM=0.7342, PSNR=20.5205; For UP: SSIM=0.72273, PSNR=21.2364. This means that our approach can resist roughly $90 \%$ of error perturbations, which clearly demonstrates the robustness of this filtering mechanism. Indeed, ACC filter improves by about $75 \%$ passing from $15 \%$ to $90 \%$. Additionally, Figure 15 shows an example of the obtained Lena image before perturbation, after perturbation and after applying the two alternative filtering mechanisms at $80 \%$ of errors. As shown from these figures, using the ACC filter allows to recover the image content even after introducing a high percentage of errors.

\subsubsection{Network Performance}

The network configuration we use is composed of two communicating nodes (i.e., a sender and a receiver). The communication range of the sender is set to $120 \mathrm{~m}$, and the distance between the two nodes is set to $100 \mathrm{~m}$. We use the energy consumption model proposed in [31]. The radio in this model dissipates $E_{\text {elec }}=50 \mathrm{~nJ} / \mathrm{bit}$ to run the transmitter or receiver circuitry and $\epsilon_{a m p}=100 \mathrm{pJ} / \mathrm{bit} / \mathrm{m}^{2}$ for the transmit amplifier. Thus, to transmit a $k$-bit message within a distance $d$ using this model, the radio consumes:

$$
E_{T x}(k, d)=E_{\text {elec }} * k+\epsilon_{\text {amp }} * k * d^{2}
$$

and to receive this message, the radio consumes:

$$
E_{R x}(k)=E_{\text {elec }} * k
$$

In order to measure the possible gain achieved by the proposed approach in term of saving consumed energy, we have proposed two scenarios for comparison: (a) using both filtering mechanisms associated with the proposed communication protocol, and (b) using the ARQ protocol with retransmissions. As previously explained, in ARQ protocol, the receiver does not allow any erroneous packet to pass to the higher layer. Instead, a retransmission of this packet is involved. We have denoted $\alpha=\{1,2,3,4,5\}$ as the number 


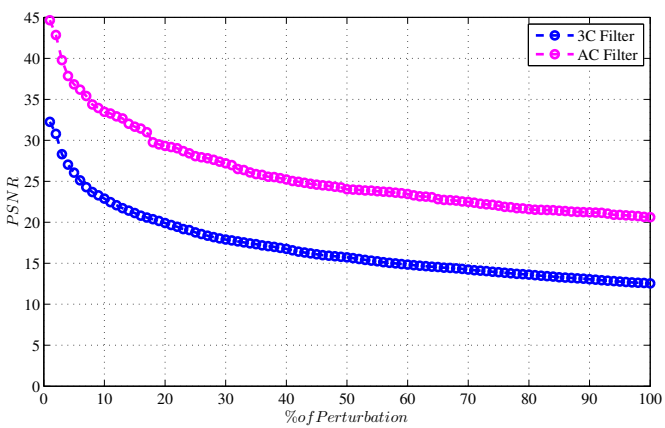

(a)

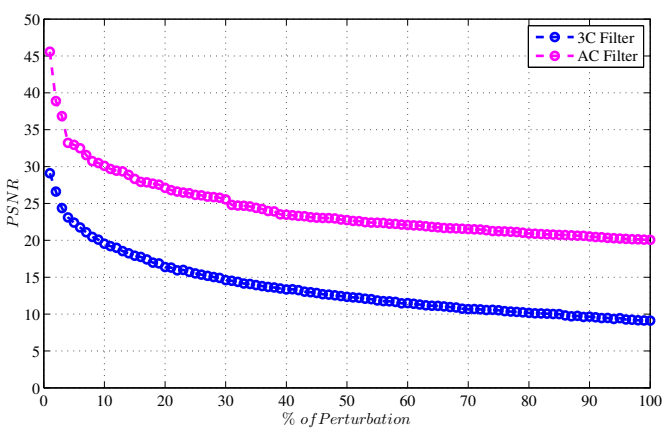

(c)

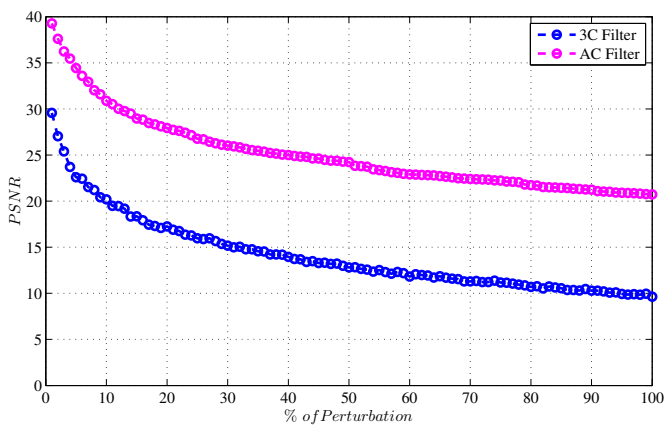

(e)

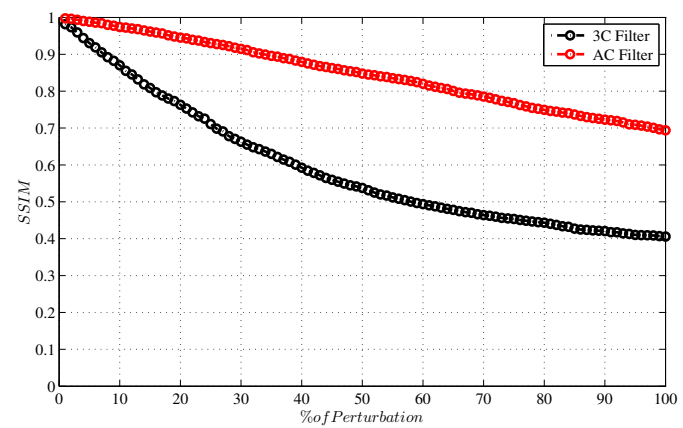

(b)

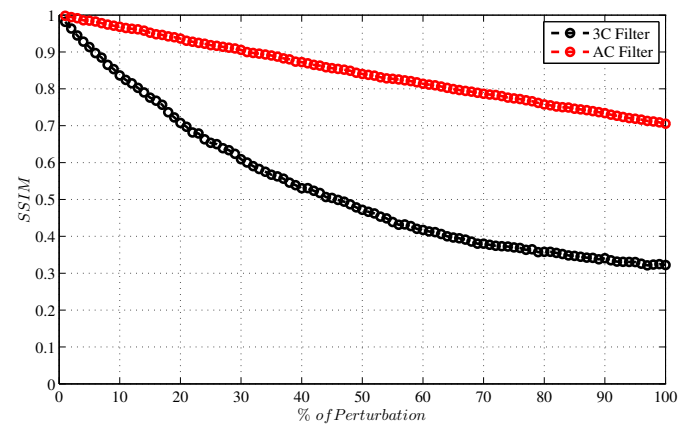

(d)

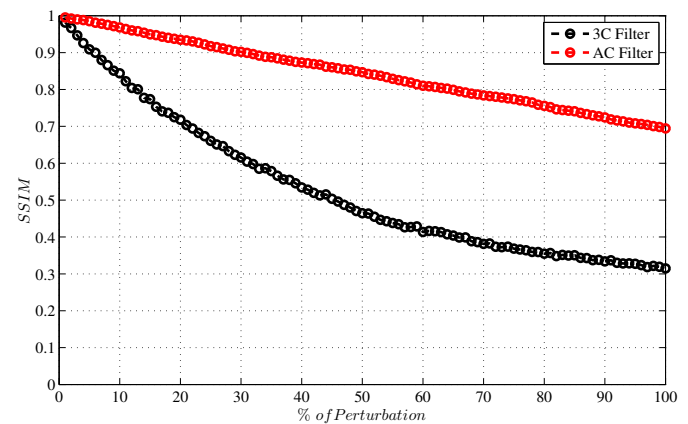

(f)

Figure 13: (a),(b)-The variation of PSNR and SSIM indexes after applying 3C and ACC filter, respectively, under Consecutive perturbation. (c),(d)-The variation of PSNR and SSIM indexes after applying 3C and ACC filter, respectively under Gaussian perturbation. (e),(f)-The variation of PSNR and SSIM indexes after applying 3C and ACC filter, respectively under Uniform perturbation.

of necessary retransmissions before success. We varied the transmission errors from $1 \%$ to $20 \%$, using the $3 \mathrm{C}$ filter (the maximum value under which this filter can defeat to errors, as previously explained) and to $90 \%$, using the ACC filter. For each scenario, we have measured the required total energy (i.e., transmission and reception). Figure 15 provides the obtained results. 


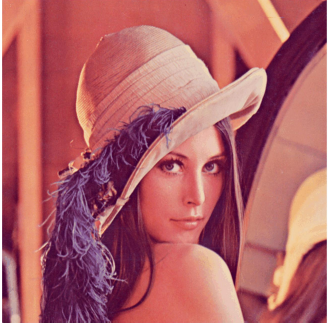

(a)

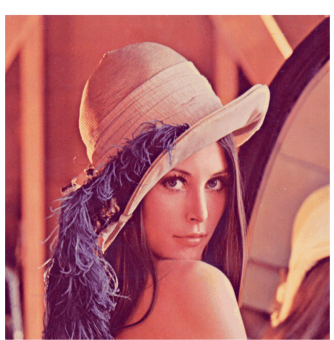

(e)

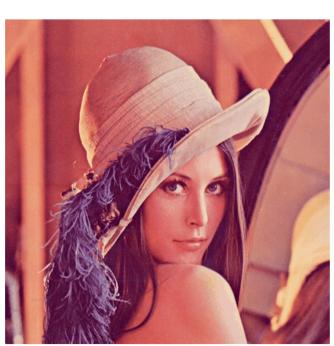

(i)

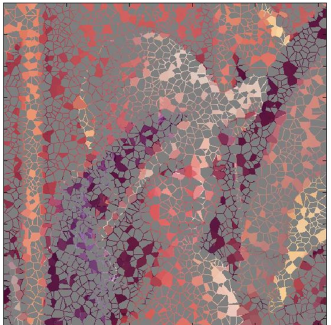

(b)

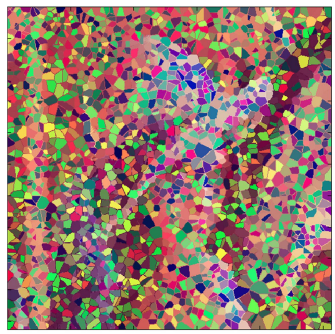

(f)

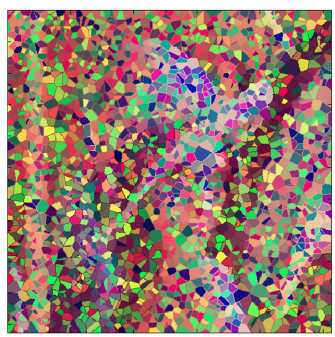

(j)

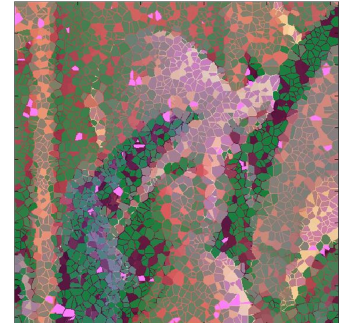

(c)

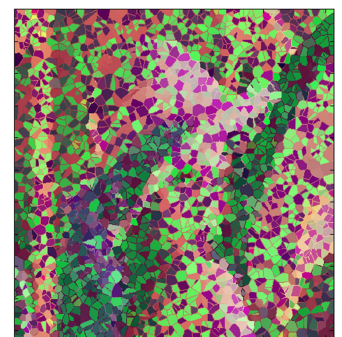

(g)

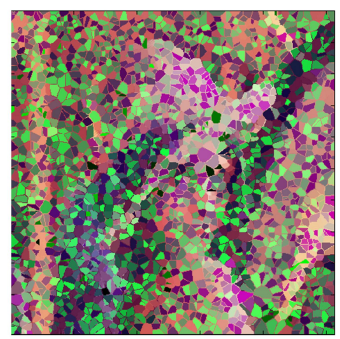

(k)

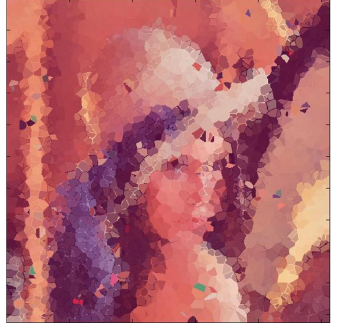

(d)

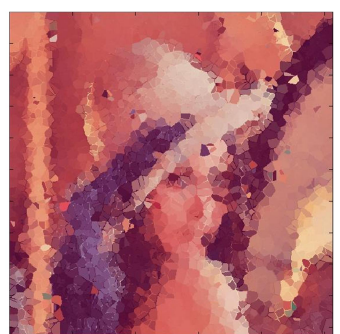

(h)

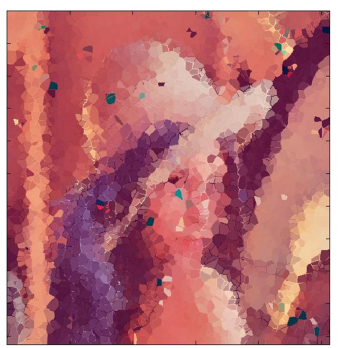

(I)

Figure 14: (a), (e), (i) Lena image before Voronoi tessellation. (b), (f),(j) Lena image after Voronoi tessellation under 90\% of CP, UP and GP, respectively. (c),(g),(k) Lena image after Voronoi tessellation and after applying the proposed 3C filtering. (d),(h),(l) Lena image after Voronoi tessellation and after applying the proposed ACC filtering.

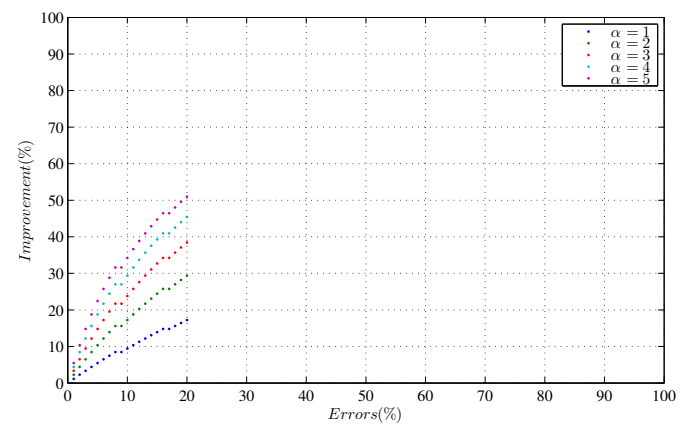

(a)

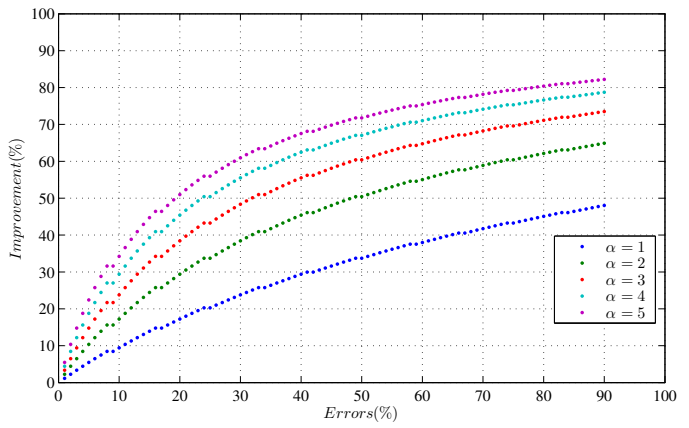

(b)

Figure 15: (a),(b) Improvement in term of energy consumption between our proposed protocol and the ARQ protocol for each $\alpha$ value within a different percentage of errors (\%) and after applying the $3 \mathrm{C}$ filter and ACC filter, respectively. 
In Figure 15-(a), where our proposed communication protocol is applied with 3C filtering mechanism, an improvement over ARQ protocol with approximately 20\% is achieved with $\alpha=1$ and $50 \%$ with $\alpha=5$. Additionally, using the second filtering technique ACC filter, an improvement of approximately $40 \%$ is achieved with one retransmission and $80 \%$ with five retransmissions as illustrated in Figure 15-(b). Overall, the gain is substantial and clearly demonstrates the effectiveness of our proposed approach to cope with transmission errors. Thus, it makes the proposed approach very suitable for dealing with constrained networks, where the destination node has sufficient resources.

\section{Conclusion}

In this paper, a novel error resilience scheme has been proposed. This approach permits the destination to participate in handling errors that may occur during transmission. To this end, a new communication protocol, inspired from ARQ protocol, has been associated to report the corrupted packets to higher layer. Besides, in order to enhance the received corrupted image, two recovering filtering mechanisms have been presented and studied. The combination of both techniques has proved that the proposed communication protocol has greatly reduced the number of retransmissions compared to the standard ARQ, with an improvement ranging from $20 \%$ to more than $80 \%$, in the overall energy consumption. Additionally, simulation results have demonstrated that using the first filtering technique (3C filter), Voronoi approach can resist to approximately $20 \%$ of errors, while using the second filtering technique, more than $90 \%$ of errors have been recovered and corrected. As a result, we can conclude that the proposed Voronoi approach can ensure data confidentiality against different types of perturbations with low complexity and minimum energy consumption.

\section{References}

[1] A. S. Wander, N. Gura, H. Eberle, V. Gupta, S. C. Shantz, Energy analysis of public-key cryptography for wireless sensor networks, in: Pervasive Computing and Communications, 2005. PerCom 2005. Third IEEE International Conference on, IEEE, 2005, pp. 324-328.

[2] D. G. Padmavathi, M. Shanmugapriya, et al., A survey of attacks, security mechanisms and challenges in wireless sensor networks, arXiv preprint arXiv:0909.0576.

[3] A. Mostefaoui, H. Noura, Z. Fawaz, An integrated multimedia data reduction and content confidentiality approach for limited networked devices, Ad Hoc Networks 32 (2015) 81-97.

[4] M. C. Vuran, I. F. Akyildiz, Error control in wireless sensor networks: a cross layer analysis, Networking, IEEE/ACM Transactions on 17 (4) (2009) 1186-1199.

[5] N. A. Flayh, R. Parveen, S. I. Ahson, Wavelet based partial image encryption, in: Multimedia, Signal Processing and Communication Technologies, 2009. IMPACT'09. International, IEEE, 2009, pp. 32-35.

[6] M. Ghadi, L. Laouamer, T. Moulahi, Securing data exchange in wireless multimedia sensor networks: perspectives and challenges, Multimedia tools and applications 75 (6) (2016) 3425-3451.

[7] E. Biham, A. Shamir, Differential cryptanalysis of the data encryption standard, Springer Science \& Business Media, 2012.

[8] J. Daemen, V. Rijmen, The design of Rijndael: AES-the advanced encryption standard, Springer Science \& Business Media, 2013.

[9] U. Somani, K. Lakhani, M. Mundra, Implementing digital signature with rsa encryption algorithm to enhance the data security of cloud in cloud computing, in: Parallel Distributed and Grid Computing (PDGC), 2010 1st International Conference on, IEEE, 2010, pp. 211-216.

[10] X. Liao, S. Lai, Q. Zhou, A novel image encryption algorithm based on self-adaptive wave transmission, Signal processing 90 (2010) 2714-2722.

[11] C.-K. Chen, C.-L. Lin, C.-T. Chiang, S.-L. Lin, Personalized information encryption using ecg signals with chaotic functions, Information Science 193 (2012) 125-140.

[12] Y. Zhou, L. Bao, C. P. Chen, Image encryption using a new parametric switching chaotic system, Signal Processing 93 (11) (2013) 3039-3052. 
[13] Z. Zhu, W. Zhang, K.-W. Wong, H. Yu, A chaos-based symmetric image encryption scheme using a bit-level permutation, Information Science 181 (6) (2011) 1171-1186.

[14] C. Guo, S. Liu, J. Sheridan, Optical double image encryption employing a pseudo image technique in the fourier domain, Opt. Commun. 321 (6) (2014) 61-72.

[15] J. Lima, L. Novaes, Image encryption based on the fractional fourier transform over finite fields, Signal Processing 94 (2014) $521-530$

[16] A. Cheddad, J. Condell, K. Curran, P. McKevitt, A hash-based image encryption algorithm, Optics communications 283 (6) (2010) 879-893.

[17] B. Norouzi, S. M. Seyedzadeh, S. Mirzakuchaki, M. R. Mosavi, A novel image encryption based on hash function with only two-round diffusion process, Multimedia systems 20 (1) (2014) 45-64.

[18] A. Jolfaei, A. Mirghadri, Survey: image encryption using salsa20, International Journal of Computer Science Issues 7 (5) (2010) 213-220.

[19] T. Xiang, J. Qu, D. Xiao, Joint spiht compression and selective encryption, Applied Soft Computing 21 (2014) 159-170.

[20] G. Gu, J. Ling, G. Xie, Z. Li, A chaotic-cipher-based packet body encryption algorithm for jpeg2000 images, Signal Processing: Image Communication 40 (2016) 52-64.

[21] I. F. Akyildiz, T. Melodia, K. R. Chowdhury, A survey on wireless multimedia sensor networks, Computer networks 51 (4) (2007) 921-960.

[22] C. Carthern, W. Wilson, R. Bedwell, N. Rivera, Data link layer, in: Cisco Networks, Springer, 2015, pp. 35-48.

[23] T. Fritz, C. Rossi, N. Yague-Martinez, F. Rodriguez-Gonzalez, M. Lachaise, H. Breit, Interferometric processing of tandem-x data, in: Geoscience and Remote Sensing Symposium (IGARSS), 2011 IEEE International, IEEE, 2011, pp. $2428-2431$.

[24] A. Fujiwara, T. Dohi, T. Sato, Coding and decoding system using crc check bit, uS Patent 6,081,919 (Jun. 27 2000).

[25] R. Braden, D. Borman, C. Partridge, Computing the internet checksum, ACM SIGCOMM Computer Communication Review 19 (2) (1989) 86-94.

[26] B. R. Hunt, R. L. Lipsman, J. M. Rosenberg, A guide to MATLAB: for beginners and experienced users, Cambridge University Press, 2014.

[27] Q. Huynh-Thu, M. Ghanbari, Scope of validity of psnr in image/video quality assessment, Electronics letters 44 (13) (2008) $800-801$

[28] Z. Wang, A. C. Bovik, H. R. Sheikh, E. P. Simoncelli, Image quality assessment: from error visibility to structural similarity, Image Processing, IEEE Transactions on 13 (4) (2004) 600-612.

[29] Z. Wang, A. C. Bovik, Mean squared error: love it or leave it? a new look at signal fidelity measures, Signal Processing Magazine, IEEE 26 (1) (2009) 98-117.

[30] V. Rani, A brief study of various noise model and filtering techniques, Journal of global research in computer science 4 (4) (2013) 166-171.

[31] W. B. Heinzelman, A. P. Chandrakasan, H. Balakrishnan, An application-specific protocol architecture for wireless microsensor networks, Wireless Communications, IEEE Transactions on 1 (4) (2002) 660-670. 